Ann. Génét. Sél. anim., I970, 2 (4), 355-36r.

\title{
ANALYSE CHROMOSOMIQUE DE DEUX PORCS INTERSEXUÉS (SUS SCROFA DOMESTICUS)
}

\author{
Françoise HULOT (*) \\ Station centrale de Génétique animale, \\ Centre national de Recherches zootechniques, 78-Jouy-en-Josas \\ Institut national de la Recherche agronomique
}

\section{RESUMÉ}

Deux porcs Large-White hermaphrodites, soumis à des analyses caryologiques sanguines, révèlent une formule chromosomique femelle normale $(36$ autosomes $+\mathrm{XX}$ ). L'étude de leur tractus, à prédominance femelle, permet de concevoir l'existence d'intersexués féconds. La dualité existant entre un système génital mixte et une constitution chromosomique $\mathrm{XX}$ est discutée : parmi les diverses possibilités, l'hypothèse génétique est particulièrement retenue, l'apparition de l'anomalie présentant une certaine fréquence.

\section{INTRODUCTION}

L'intersexualité chez le Porc est fréquente et s'accompagne le plus souvent d'un pseudo-hermaphrodisme mâle ou d'un hermaphrodisme vrai. Les études caryologiques ont démontré la constitution génétique femelle de la plupart de ces intersexués. Cependant, chez un petit nombre d'animaux, des formules chromosomiques variées ont été signalées : caryotype mâle normal (syndrome du testicule féminisant), chimère $\mathrm{XX} / \mathrm{XY}$ (freemartinisme), trisomie des hétérosomes (XXY, syndrome de Klinefelter) (cf. HuLOT I969, pour revue).

Ce travail a pour but de décrire l'anatomie et de définir le sexe chromosomique de deux porcs intersexués.

$(*)$ Nouvelle adresse : Institut national de la Recherche agronomique, Centre de Recherches de Toulouse, Laboratoire de Méthodologie génétique, Chemin de Borde Rouge Auzeville, B. P. I 2, 3I-Castanet-Tolosan. 


\section{MATÉRIEL, ETT TECHNIQUES}

Ces intersexués, de race Large-White, proviennent du troupeau expérimental du Domaine de Galle (18-Avord). Leur filiation est connue, et ils ne sont pas apparentés. L'existence de l'anomalie a été constatée entre 6 et 7 mois, époque vers laquelle ces animaux ont présenté un comportement à tendance mâle.

Leur morphologie génitale est femelle; ils présentent cependant un clitoris hypertrophié et un orifice vulvaire de forme allongée.

Le tractus génital a été prélevé à l'abattage puis photographié et soumis à un examen descriptif.

Des cultures de sang ont été réalisées selon la microtechnique de DE Grouchy et al. (1964). Les milieux de culture utilisés, 199 ou NCTC, en présence du sérum de l'animal ou de sérum de veau, ont donné d'excellents résultats, la croissance cellulaire étant active et le nombre des mitoses élevé (plus de 100 métaphases par lames dans les meilleures conditions).

La détermination du sexe a été basée sur la présence ou l'absence du chromosome $Y$, métacentrique de taille réduite, relativement aisé à identifier à l'examen microscopique, et non pas sur celle de l'hétérosome $\mathrm{X}$ dont la morphologie et la taille, proches de celles de certains autosomes, ne permettent pas de reconnaissance sexuelle immédiate.

Un tiers environ des cellules examinées a été photographié pour procéder à un comptage chromosomique et une dizaine de clichés ont servi à établir le caryotype des animaux.

Nous nous sommes référés au schéma classique proposé par McFEeLY et HARE (1966) en y apportant toutefois de légères modifications. En particulier, nous n'isolons pas la paire de chromosomes $\mathrm{X}$ du groupe $\mathrm{C}$, car son identification rigoureuse nous parait, comme à de nombreux auteurs, contestable en l'absence de critères précis (mesures biométriques, examens autoradiographiques par exemple).

\section{RÉSULTATS}

\section{I. - Anatomie macroscopique du tractus génital}

Les caractéristiques du tractus des deux animaux sont rapportées dans le tableau $I$ et la figure $I$.

TABLEAU I

Anatomie du tractus génital

\begin{tabular}{|c|c|c|c|}
\hline \multicolumn{2}{|c|}{ Intersexué (I) } & \multicolumn{2}{|c|}{ Intersexué (II) } \\
\hline Côté droit & Côté gauche & Côté droit & Côté gauche \\
\hline $\begin{array}{l}\text { ovaire avec follicules } \\
\text { hypertrophiés } \\
\text { testicule atrophié } \\
\text { épididyme } \\
\text { canal déférent } \\
\text { oviducte } \\
\text { corne utérine réduite }\end{array}$ & $\begin{array}{l}\text { ovaire atrophié } \\
\text { testicule atrophié } \\
\text { épididyme } \\
\text { canal déférent } \\
\text { corne utérine }\end{array}$ & $\begin{array}{l}\text { testicule atrophié } \\
\text { épididyme } \\
\text { canal déférent } \\
\text { oviducte atrophié } \\
\text { corne utérine réduite }\end{array}$ & $\begin{array}{l}\text { ovaire avec follicules } \\
\text { hypertrophiés } \\
\text { testicule très atrophié } \\
\text { épididyme } \\
\text { canal déférent } \\
\text { oviducte } \\
\text { corne utérine }\end{array}$ \\
\hline $\begin{array}{rr}r & \text { corps } \\
\text { vulve } & \begin{array}{c}\text { vallongée } \\
\text {-clitoris hy }\end{array}\end{array}$ & $\begin{array}{l}\text { utérin } \\
\text { gin } \\
\text { vec méat urinaire } \\
\text { pertrophié }\end{array}$ & $\begin{array}{l}\text { corps utérin court } \\
\text { vagin }\end{array}$ & $\begin{array}{l}\text { rin court } \\
\text { gin } \\
\text { rec méat urinaire } \\
\text { pertrophié }\end{array}$ \\
\hline
\end{tabular}




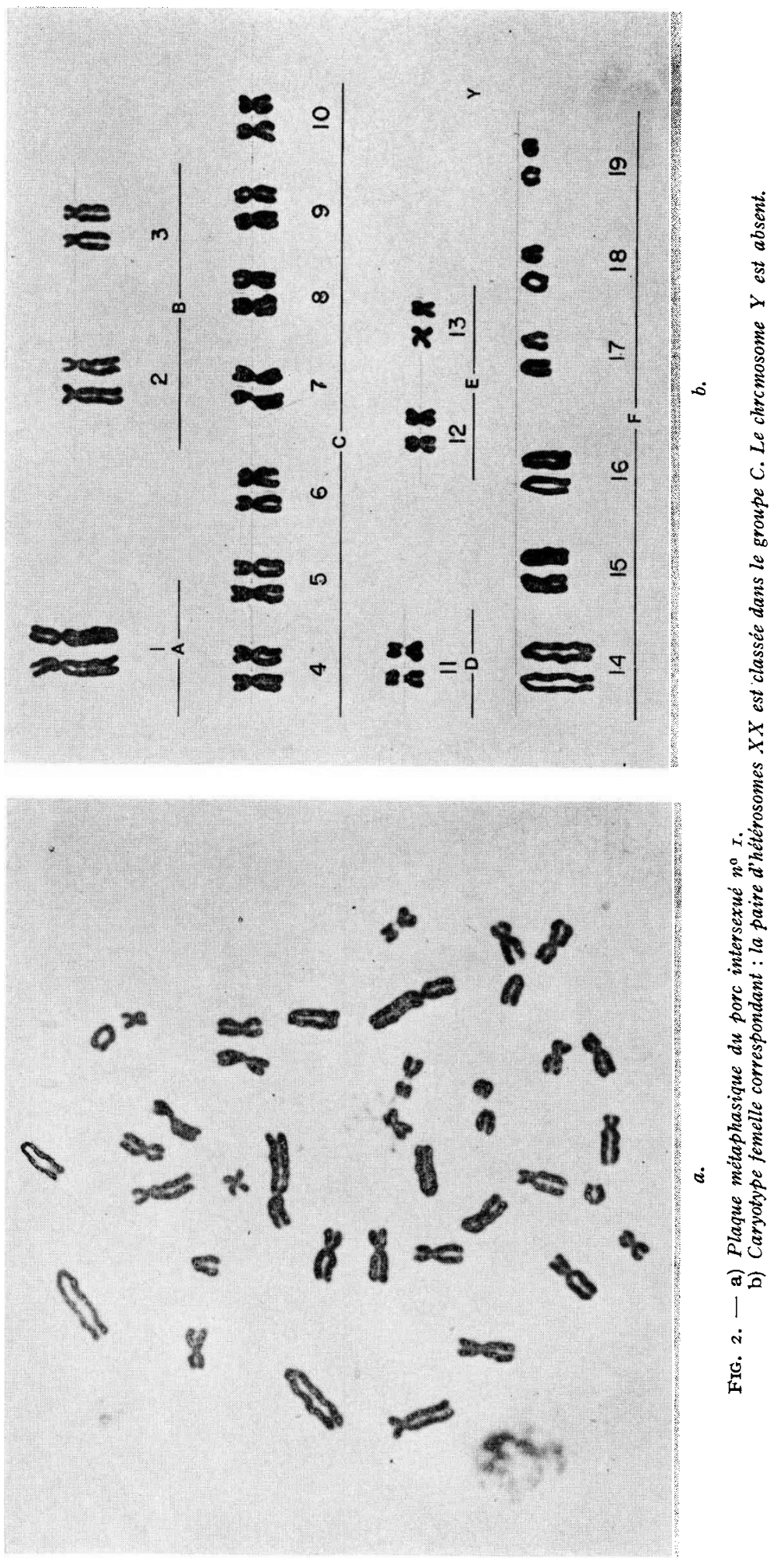


Ils présentent une juxtaposition de structures mâles et femelles. La partie ovarienne montre des follicules kystiques hypertrophiés, notamment chez l'animal II. Dans les deux cas, les cornes utérines sont nettement dissymétriques : la corne atrophiée est du côté de l'ovaire hypertrophié chez l'animal I, du côté testiculaire chez l'animal II.

Ces intersexués sont des hermaphrodites vrais.

\section{2. - Examen caryologique des leucocytes}

Les résultats sont donnés dans le tableau 2.

TABLEAU 2

Examen des métaphases

\begin{tabular}{|c|c|c|c|c|c|c|c|}
\hline \multirow{3}{*}{ Animaux } & \multicolumn{2}{|c|}{$\begin{array}{c}\text { Examens } \\
\text { au microscope }\end{array}$} & \multicolumn{3}{|c|}{$\begin{array}{c}\text { Examens } \\
\text { sur photographies }\end{array}$} & \multicolumn{2}{|c|}{ Caryotype } \\
\hline & \multirow{2}{*}{$\begin{array}{l}\text { Nombre } \\
\text { de cellules } \\
\text { exami- } \\
\text { nées }\end{array}$} & \multirow{2}{*}{$\begin{array}{l}\text { Sexe } \\
\text { gonoso- } \\
\text { mique }\end{array}$} & \multirow{2}{*}{$\begin{array}{c}\text { Nombre } \\
\text { de cellules } \\
\text { exami- } \\
\text { nées }\end{array}$} & \multicolumn{2}{|c|}{$\begin{array}{c}\text { Nombre } \\
\text { chromosomique }\end{array}$} & \multirow{2}{*}{$\begin{array}{c}\text { Nombre } \\
\text { de cellules } \\
\text { exami- } \\
\text { nées }\end{array}$} & \multirow{2}{*}{$\begin{array}{c}\text { Anoma- } \\
\text { lies }\end{array}$} \\
\hline & & & & $2 n=38$ & $2 n \# 3^{8}$ & & \\
\hline I & 325 & $\mathrm{xx}$ & II 5 & 97 & I 8 & 5 & $\circ$ \\
\hline II & I 74 & $\mathbf{x x}$ & 62 & 55 & 7 & 5 & 0 \\
\hline
\end{tabular}

Chez les deux animaux, toutes les cellules examinées sont exemptes de l'hétérosome Y; $85 \mathrm{p}$. Ioo et $88 \mathrm{p}$. Ioo d'entre elles, pour les animaux I et II respectivement, ont un nombre chromosomique normal $(2 n=38)$. Le grand nombre de cellules examinées conduit à penser que la constitution chromosomique du tissu sanguin est $\mathrm{XX}$ de façon homogène, et que la probabilité d'existence d'une chimère $\mathrm{XX} / \mathrm{XY}$ est faible.

Par ailleurs, aucune anomalie autosomale n'a été remarquée (fig. 2).

\section{DISCUSSION-CONCLUSION}

La féminisation prépondérante de l'ensemble du système génital de ces deux animaux intersexués est à noter. Le développement de l'ovaire, la longueur à peu près normale de l'une des cornes utérines, la constitution chromosomique $\mathrm{XX}$ des animaux, sont des éléments qui, considérés isolément, sont compatibles avec l'activité reproductrice. Bien que le niveau de féminisation observé ne permette pas de prétendre que ces deux animaux auraient pu être fertiles, il n'est pas exclu 
que des intersexués porteurs d'un système génital femelle plus proche de la normale, puissent être féconds. Ceci a d'ailleurs été observé (FoLGERS, I932; WII.liams, I943; Perry et Pomeroy, I956; Smidt, I962; Hulland, I964; Benort, r964; Cox I968; Logstestrun, I968. Dans tous les cas d'intersexualité décrits, lorsqu'il y a fertilité, seul le système reproducteur femelle est fonctionnel.

L'origine de la dualité existant entre un système génital mixte et une constitution chromosomique femelle n'est pas élucidée. Certains auteurs l'expliquent en admettant la possibilité d'une translocation partielle de $\mathrm{Y}$ sur $\mathrm{X}$ (FERGUSONSmIDT, I966) ou l'existence d'une double population cellulaire XX/XY, cette dernière étant très faiblement représentée. Les techniques caryologiques sont relativement grossières et leurs résultats ne permettent pas d'exclure ces hypothèses. D'autres auteurs (MCFEELY et al., I967; HAMERTON, I968) ont suggéré un mécanisme génétique qui pourrait se concevoir ainsi : 1'hétérosome $\mathrm{X}$ serait porteur de gènes mâles normalement réprimés chez la femelle; une dérépression de ces gènes pourrait être induite soit par une mutation, soit par un " modificateur autosomique " entraînant ainsi la masculinisation partielle d'individus XX. Chez la chèvre motte d'origine alpine par exemple, l'existence d'un tel modificateur autosomique est connue : le gène $\mathrm{P}$ 《 motte ", à l'état homozygote, transforme les femelles en intersexuées (Jost et LAUVERGNE, I969). Un mécanisme du même type pourrait exister chez le Porc (HAMERTon, I968).

La fréquence des intersexués dans la race Large-White étudiée est de 1'ordre de I p. roo. Cette constante peut être une présomption en faveur de 1'hypothèse génétique. Des enquêtes étendues et des accouplements dirigés seraient nécessaires pour en vérifier le bien-fondé. A l'appui de cette suggestion, il faut noter que de nombreux auteurs (Косн et al., I957. JoHNSTON et al., I958, GERNEKE, r 967 , pour revue) sont convaincus de la nature héréditaire de cette anomalie, rencontrée dans plusieurs races porcines et pouvant atteindre jusqu'à $20 \mathrm{p}$. Ioo des animaux de certains troupeaux isolés (PoND et al. I96I). Le contrôle de l'apparition des porcs intersexués échappe actuellement au généticien. L'absence d'un effet conjoint " marqueur " tel l'absence de corne chez la chèvre, rend l'étude de ce caractère malaisée dans l'espèce porcine. De plus, la faible incidence économique de cette aberration est probablement à l'origine du peu d'intérêt qui lui a été porté jusqu'alors.

Reçu pour publication en juin 1970.

\section{REMERCIEMENTS}

Je remercie Mme Ondine Bomsel-Helmreich des conseils et suggestions qu'elle a bien voulu me proposer. 


\section{SUMMARY}

\section{Chromosomic anALysis OF TWO INTERSEXED PIGS (SUS SCROFA DOMESTICUS)}

Caryologic blood analyses of two hermaphroditic Large-White pigs showed a normal female chromosome formula (36 autosomes $+\mathrm{XX}$ ).

Study of their predominately female tracts leads us to believe that fertile intersexed individuals may exist. The duality of a mixed genital system and an XX chromosomic constitution is discussed. Among various possibilities, a genetic hypothesis seems most likely since this abnormality occurs with some frequency.

\section{REFERENCES BIBLIOGRAPHIQUES}

BENoIT R., 1964. Quelques considérations sur l'hermaphrodisme. Schweizer Arch. Tierheilk., 106, $63-73$. Cox J. E., 1968. A case of a fertile intersex pig (abstr.). J. Reprod. Fert., 16, 321-322.

FERGUSON-Smith M. A., I966. X-Y chromosomal interchange in the aetiology of true hermaphroditism and of XX klinefelter's syndrome. Lancet, ii, 475-476.

Folgers A. F., 1932. A case of hermaphroditism with pregnancy in a pig. Acta path. microbiol. scand.1 supplem. 11, I43.

GerNeKE W. H., I967. Cytogenetic investigations on normal and malformed animals, with specia, reference to intersexes. Ondersteport J. vet. Res., 34, 219-299.

Grouchy J. DE, Roubin M., Passage E., I964. Microtechnique pour l'étude des chromosomes humains à partir d'une culture de leucocytes sanguins. Annls Génét., 7, 25-30.

Hamerton J. L., 1968. Significance of sex chromosome derived heterochromatin in Mammals. Nature, 219, 9 ro-914.

Hulland I. J., I964. Pregnancy in a hermaphrodite sow. Can. vet. J., 5, 39.

Hulot Françoise, 1969. Les chromosomes des Suiformes. Annls Génét. Sél. anim., 1, 315-336.

Johnston E. F., Zeller J. H., Cantwell G., 1958. Sex anomalies in swine. J. Hered., 49, $255-26 \mathrm{r}$.

Jost A., I.AuVERGNe J. J., r969. Quelques réflexions à l'issue du Séminaire sur l'intersexualité chez la chèvre sans cornes. Annls Génét. Sél. anim., 1, 465-468.

Koch P., FIscher H., Schumann H., 1957. Erbpathologie der landwirtschaftlichen Haustiere. Berlin, Paul Parey, r67-170.

Logtestijn C. Y. Van, r968. Pregnancy in the hermaphrodite sow. (Neerl.) rés. ang1. Tijdschr. Diergeneesk., 93, 523-525.

McFeely R. A., Hare W. C. D., 1966. Cytogenetic studies of the domestic pig. In L. K. Bustad, Swine in biomedical research, 13-24. Frayne Printing, Seattle.

McFeely R. A., Hare W. C. D., Biggers J. D., 1967. Chromosomes studies in 44 cases of intersex in domestic Mammals. Cytogenetics, 6, 242-253.

Perry J. S., Pomeroy R. W., 1956. Abnormalities of the reproductive tract of the sow. J. agric. Sci. Camb., 47, 238.

Pond W. G., Roberts S. J., Simmons K. R., 1961. True and pseudo-hermaphroditism in swine herd. Cornell vet., 51, 394-404.

Scofield A. M., Cooper K. J., LAMming G. E., 1969. The distribution of embryos in intersex pigs. J. Reprod. Fert., 20, 16r-164.

SMmt D., I962. Einige Beobachtungen über das Vorkommen von Zwittern beim Schwein. Zuchthyg. Fortpfliör Besam. Haustiere, 6, 371.

WILliams W. I., I943. Diseases of the genital organs of domestic animals. 3rd ed. Plimpton, Mass. 